ఠ

\title{
Role of colesevelam in managing heterozygous familial hypercholesterolemia in adolescents and children
}

REVIEW

\author{
Travis Sonnett \\ Jennifer Robinson \\ Paul Milani \\ R Keith Campbell \\ Department of Pharmacotherapy, \\ College of Pharmacy, Washington \\ State University, Pullman, Washington, \\ USA
}

This article was published in the following Dove Press journal:

Adolescent Health, Medicine and Therapeutics

29 July 2010

Number of times this article has been viewed
Background: Colesevelam hydrochloride is a synthetic, nonsystemically absorbed polymer that functions as a bile acid sequestrant for the treatment of hypercholesterolemia. Recently, colesevelam was investigated for the treatment of heterozygous familial hypercholesterolemia $(\mathrm{HeFH})$ in the pediatric/adolescent population aged 10-17 years.

Objective: The purpose of this article is to review the disease state of $\mathrm{HeFH}$ in children and adolescents, review the pharmacologic mechanism of action, kinetics, and safety profile of colesevelam, analyze the results of a recent clinical trial of colesevelam in the pediatric/adolescent $\mathrm{HeFH}$ population, and discuss the role of colesevelam as a viable treatment option for HeFH. Methods: A literature search using Medline (1966-03 May 2010), PubMed (1950-03 May 2010), Science Direct (1994-03 May 2010), and International Pharmaceutical Abstracts (2004-2010) was performed using the search term colesevelam. English language, original research, and review articles were examined, and citations from these articles were also assessed. The manufacturer's prescribing information and the Food and Drug Administration review of the new drug application for the powder formulation were also examined.

Results: A 32-week trial was performed investigating the efficacy of colesevelam as monotherapy or combination therapy with a stable statin regimen. Upon completion of the trial, significant benefits were found in regard to the treatment of $\mathrm{HeFH}$ and the lowering of low-density lipoprotein cholesterol, total cholesterol, and other secondary measures. Safety and tolerability were also examined throughout the duration of the clinical trial, with adverse drug reactions considered mild in severity.

Conclusion: Colesevelam has been shown to reduce low-density lipoprotein cholesterol levels significantly in pediatric/adolescent patients with $\mathrm{HeFH}$, while maintaining a mild side effect profile. Although further research would be beneficial for long-term effects in this population, colesevelam should be considered when developing a treatment regimen for $\mathrm{HeFH}$ in the pediatric/adolescent population.

Keywords: colesevelam, heterozygous familial hypercholesterolemia, bile acid sequestrant, hyperlipidemia, pediatric

\section{Introduction}

Diabetes mellitus, cardiovascular disease (CVD), and dyslipidemia are growing concerns in children and adolescents worldwide, as the reported numbers of youth affected by one or more of these disease states continue to rise. As evidence of this increasing burden on the health of US children, a 2008 prescription use study of chronic medication therapy reported an increase of $15 \%$ for lipid-lowering medications. ${ }^{1}$ Early development of atherosclerotic lesions in children and adolescents has also been associated with higher total cholesterol count (TC), higher low-density lipoprotein (LDL), and lower levels of
Correspondence:Travis Sonnett

PO Box 6465I0, Pullman,

WA 99164-6510, USA

Tel +I 5093358366

Fax + I 5093350162

Email tsonnett@wsu.edu 
high-density lipoprotein (HDL). ${ }^{2}$ Related risk factors, such as dyslipidemia, smoking, and obesity, have also been found to be a significant determinant of the extent of atherosclerotic plaque formation in children and adolescents. ${ }^{3}$ In 2008, the American Academy of Pediatrics released clinical guidelines outlining the recommended protocol for screening and treatment of lipid abnormalities in children and adolescents. ${ }^{4}$ These guidelines called for the screening of any child age two years and older with a family history of dyslipidemia or early heart disease, and those with other cardiovascular risk factors, such as obesity, hypertension, diabetes mellitus, or smoking. While initial therapy for a reduction in TC may involve diet and/or exercise, patients at greater risk of negative outcomes may be candidates for pharmacotherapy. The American Academy of Pediatrics guidelines call for a consideration of therapy when a patient (eight years or older) has LDL $>190 \mathrm{mg} / \mathrm{dL}$ with no other risk factors for CVD, LDL $>160 \mathrm{mg} / \mathrm{dL}$ with other CVD risk factors present, or LDL $>130 \mathrm{mg} / \mathrm{dL}$ in combination with diabetes mellitus. Several medications for treatment of dyslipidemia in children have been investigated, including statins and bile acid binding resins. Of recent interest, colesevelam has been investigated in a small trial for use in children and adolescents with heterozygous familial hypercholesterolemia (HeFH). With its recent powder formulation gaining Food and Drug Administration (FDA) approval in the US, colesevelam poses an interesting choice in the treatment of dyslipidemic disorders in children and adolescents. This article will focus on the major dyslipidemic issues in this at-risk population, the recent study of colesevelam and its proposed place in therapy, and any issues or concerns regarding the use of colesevelam in children or adolescents.

\section{Epidemiology}

Hypercholesterolemia is defined as high blood levels of TC and LDL cholesterol, and may be accompanied by low levels of HDL cholesterol. Knowledge of cholesterol levels in children is important because they could strongly predict the risk of potential CVD in adulthood. ${ }^{5,6}$

One prominent form of hypercholesterolemia diagnosed in children is $\mathrm{HeFH}$ which affects about one in 500 people. ${ }^{7} \mathrm{HeFH}$, a genetic disorder in the LDL receptor, is characterized by abnormally high levels of LDL cholesterol. ${ }^{8,9}$ In these patients, LDL receptor-mediated absorption of plasma LDL in the liver is insufficient, leading to high blood LDL cholesterol levels.

Although hypercholesterolemia alone is asymptomatic, if it goes untreated, it can manifest as disease states that could severely hinder a patient's quality of life. Hypercholesterolemia is strongly related to aortic and coronary artery fatty streaks.
Even though these fatty streaks are harmless and reversible, they can progress into atherosclerosis in early adulthood and later manifest as stroke, transient ischemic attack, myocardial infarction, peripheral arterial disease, intermittent claudication, and limb ischemia. ${ }^{2,10-13}$

In those with familial hypercholesterolemia, the atherosclerotic process is even more accelerated, and could lead to a form of CVD before the age of 30 in those with homozygous familial hypercholesterolemia $(\mathrm{HoFH})$ and tendinous xanthoma in those with HeFH. ${ }^{14-16}$ Those with HoFH may require even more urgent attention, because one study has shown that serum LDL cholesterol levels in this population are twice as high as in those with HeFH. ${ }^{17}$ Hypercholesterolemia is also responsible for changes in the arterial wall, reduced flow-mediated dilation, and enhanced thickness of the intima media. ${ }^{18}$ These abnormal changes are recognized as a predictive measure of clinical coronary events in patients who are categorically too young for coronary events. ${ }^{19}$

\section{Current treatments}

Treatments for hypercholesterolemia are sought in pediatric patients because of the possibility of reversing hypercholesterolemia-associated damage in the childhood years. Lifestyle modifications, such as eating a sensible diet that includes low saturated fats and trans-fats, along with appropriate amounts of fiber, fruits, vegetables, whole grains, and low-fat dairy products before initiating drug therapy are among first lines of therapy for treating hypercholesterolemia at any age. ${ }^{7,20}$ However, lifestyle modifications may not always be a sufficient treatment.

Pharmacologic interventions, in addition to lifestyle modifications, may provide even greater benefits in preventing hypercholesterolemia complications because pharmacologic treatment in children with hypercholesterolemia significantly slows progression of carotid intima media thickening. ${ }^{21}$ Furthermore, angiographic trials have demonstrated that cholesterol-lowering treatments stabilize atherosclerotic plaques, while preventing formation of new lesions. ${ }^{22}$ Hydroxymethylglutaryl-coenzyme A (HMG-CoA) reductase inhibitors, better known as statins, are the main class of drugs currently used to treat pediatric $\mathrm{HeFH}$, with demonstrated safety and benefits in this population. ${ }^{8}$ In children with $\mathrm{HeFH}$, statins can reduce serum LDL cholesterol levels by $23 \%-40 \%{ }^{23}$

Another class of drugs used to treat hypercholesterolemia is bile acid sequestrants (BAS). Monotherapy with colesevelam (Welchol ${ }^{\circledR}$, Daiichi-Sankyo, Parsippany, NJ, USA), one of this family of drugs, has been shown to reduce LDL 
cholesterol levels in adults by about $15 \%-48 \%$ when used concomitantly with a statin. ${ }^{24-27}$ Colesevelam is currently approved by the US FDA for treatment of HeFH in children and adolescent patients, with one study showing it can significantly lower LDL cholesterol.

\section{Pharmacology}

Colesevelam hydrochloride is a nonabsorbable poly allylamine hydrochloride that is cross-linked with epichlorohydrin, alkylated with 1-bromodecane and 6-bromohexyltrimethylammonium bromide. The official chemical nomenclature is allylamine polymer with 1-chloro-2,3-epoxypropane, [6-(allylamino)-hexyl]trimethyl-ammonium chloride and $N$-allyldecylamine, hydrochloride (see Figure 1). ${ }^{28}$

\section{Mechanism of action}

Colesevelam hydrochloride forms a nonabsorbable complex when exposed to bile acids in the gastrointestinal tract, decreasing enterohepatic bile acid recirculation, and increasing the excretion of bile acid salts in the feces. ${ }^{28}$ The resulting reduction in recycled bile acids increases the activity of $7 \alpha$-hydroxylase, a key enzyme responsible for the conversion of intracellular cholesterol to bile acids. ${ }^{29}$ The shift in utilization of intracellular cholesterol results in a direct decrease in production of LDL, and a resultant decrease in plasma LDL cholesterol levels and TC levels. ${ }^{30}$ The decrease in intracellular cholesterol stimulates increased hepatic production of cholesterol through the activity of the HMG-CoA reductase pathway, leading to increased cholesterol synthesis. While thought to be a factor in raising TC levels, this mechanism has not been found to increase TC, because an increase in hepatic LDL receptors along with an increase in the conversion of cholesterol to bile acids negates any increase that could take place. ${ }^{29}$ Colesevelam also increases the activity of phosphatidic acid phosphatase, and increased activity of this enzyme leads to a direct increase in the production of triglycerides. ${ }^{31}$ Disruption of enterohepatic bile acid recycling leads to an increase in phosphatidic acid phosphatase, a 10\%-14\% increase in

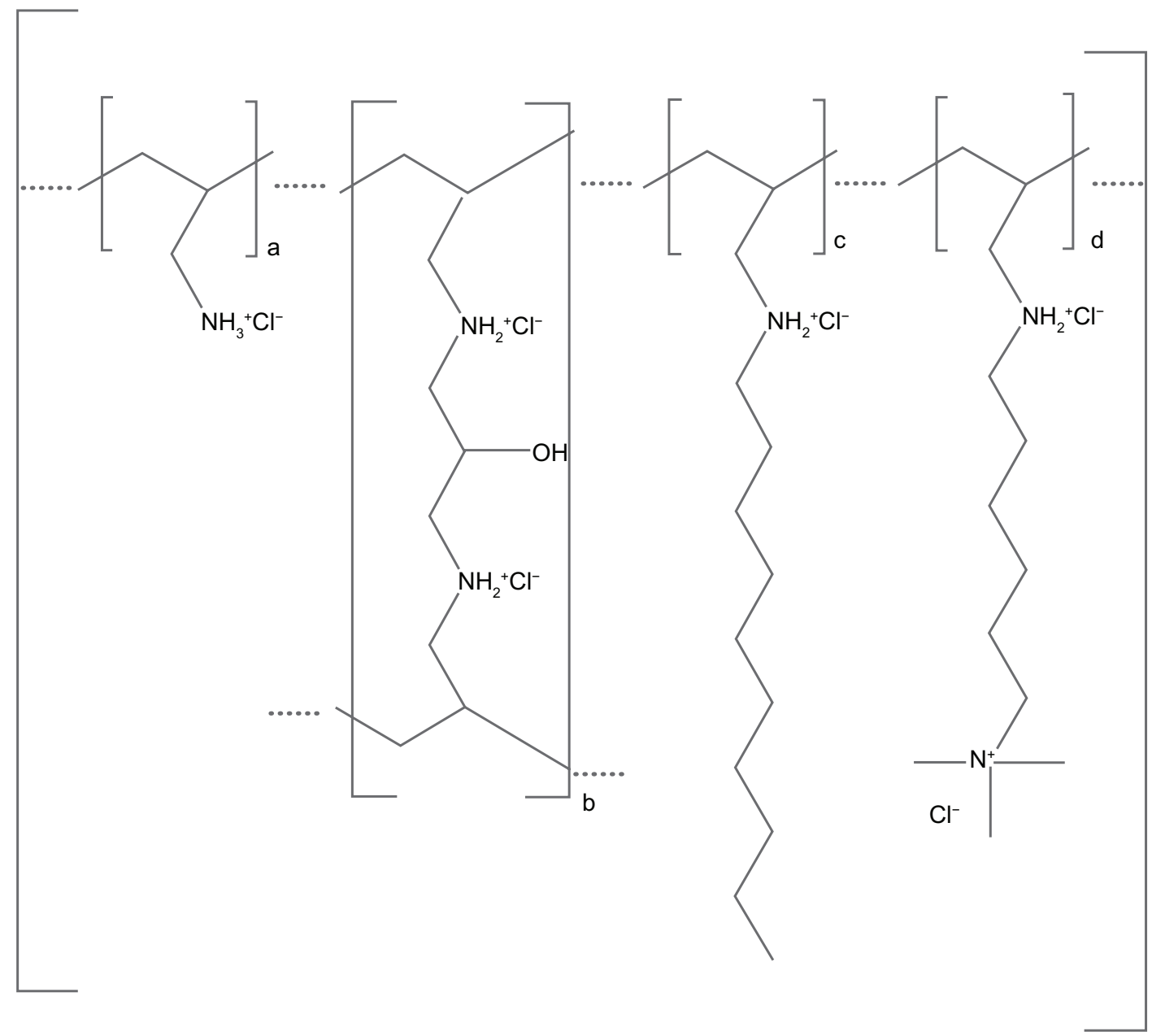

Figure I Chemical structure of colesevelam hydrochloride. ${ }^{28}$ 
plasma triglycerides, and an increase in triglyceride-rich, very low-density lipoproteins. ${ }^{25,26}$

\section{Pharmacokinetics}

The pharmacokinetic properties of colesevelam have not been studied directly in a pediatric or adolescent population. Kinetic studies performed in healthy adult volunteers have shown that colesevelam is a hydrophilic, insoluble polymer with minimal systemic absorption and distribution. ${ }^{32}$ After administration of a single dose of $\left[{ }^{14} \mathrm{C}\right]$-colesevelam to 16 healthy adult volunteers after receiving 28 days of colesevelam $1.9 \mathrm{~g}$ twice daily, the maximum detectable plasma concentration 72 hours after administration was $0.04 \%$, consistent with residual $\left[{ }^{14} \mathrm{C}\right]$. Total urine recovery of $\left[{ }^{14} \mathrm{C}\right]$-colesevelam was found to be $0 \%$, while fecal recovery accounted for $82 \%$ after a period of 192-216 hours. Animal studies in rat and canine models have also found that oral administration of colesevelam results in a plasma colesevelam level of $<1 \%$, while $98 \%$ of administered colesevelam is recovered in feces after 48-72 hours. ${ }^{33}$

Colesevelam is not systemically absorbed and, as such, factors such as distribution, protein binding sites, and metabolism are not given consideration when considering administering the medication to a patient. However, intestinal transit time does factor into consideration when considering colesevelam use; once- or twice-daily dosing options may be considered according to a patient's projected transit time. ${ }^{28}$ Colesevelam binding to bile acid increases significantly when given with food, as a result of saturation of bile acid binding sites through increased bile acid exposure. ${ }^{34}$

Future kinetic studies of colesevelam in pediatric and adolescent populations are warranted to ensure that the kinetic profile in the child/adolescent population mirrors that reported in human adult and animal models. While colesevelam has been approved by the FDA for treatment of HeFH in patients aged 10-17 years, no data are available that report the same kinetic profile in children/adolescents as in adults. Further study is appropriate to ensure a continuum of kinetic properties from adolescence to adulthood.

\section{Clinical investigation}

A study has been performed that evaluated the impact of colesevelam in pediatric/adolescent patients diagnosed with HeFH. ${ }^{35}$ Stein et al completed a 32-week randomized, double-blind, placebo-controlled, parallel-group study investigating the efficacy and safety of colesevelam or colesevelam in combination with stable statin therapy (atorvastatin, lovastatin, pravastatin, simvastatin) in pediatric subjects diagnosed with HeFH. The study was conducted in three periods. Period I, or the wash-out period established as four weeks prior to study initiation, required subjects to follow the National Cholesterol Education Program (NCEP) Step 1 diet, as well as take six placebo pills per day as a single dose or divided dose twice daily with noon and evening meals. Period II was the double-blind, randomized eight-week period, in which patients were randomized to one of three treatment groups, ie, a placebo group, a colesevelam $1.875 \mathrm{~g} /$ day group, or a colesevelam $3.75 \mathrm{~g}$ /day group. Period III was the open-label period, in which all patients received colesevelam $3.75 \mathrm{~g}$ /day as monotherapy or in addition to stable statin therapy if previously initiated, to evaluate both efficacy and safety (see Figure 1). Inclusion criteria were either LDL cholesterol $>160 \mathrm{mg} / \mathrm{dL}$ on a stable NCEP Step 1 diet for $\geq 4$ weeks and naïve to lipid-lowering therapy, or LDL cholesterol $>130 \mathrm{mg} / \mathrm{dL}$ on a stable NCEP Step 1 diet for $\geq 6$ weeks in addition to current statin therapy. Subjects were also required to have a diagnosis of $\mathrm{HeFH}$ in addition to one or more of the following: a history or presence of tendinous xanthoma or premature corneal arcus in the subject or a first-degree relative; a first-degree relative with a diagnosis of $\mathrm{HeFH}$; an untreated LDL cholesterol level of $>190 \mathrm{mg} / \mathrm{dL}$ in a first-degree relative; an untreated LDL cholesterol level of $>160 \mathrm{mg} / \mathrm{dL}$ in a sibling $<18$ years of age; premature coronary artery disease or sudden death from natural causes (male $<55$ years, female $<60$ years) in a first-degree relative. Subjects were required to have triglyceride levels $<250 \mathrm{mg} / \mathrm{dL}$, be at Tanner Stage 2 or greater, and maintain treatment compliance of $\geq 75 \%$ with the placebo washout during Period I of the study. Exclusion criteria included secondary hyperlipidemia, fasting triglyceride levels $\geq 250 \mathrm{mg} / \mathrm{dL}$, HoFH, dysphagia, dysmotility of the esophagus or bowel, hypertension, untreated thyroid disorder, uncontrolled or poorly controlled diabetes mellitus $\left(\mathrm{A}_{1 \mathrm{c}}>9 \%\right)$, pregnancy, CVD requiring immediate intervention/lipid therapy outside of the study scope, persistent elevation (three-fold) of liver function tests, and elevations in creatinine phosphokinase (10-fold) without symptoms or elevations (five-fold) with symptoms. Any medication that was deemed to interfere with the efficacy or safety evaluation was not allowed during the study period. This included, but was not limited to, all lipid-lowering agents or over-the-counter treatments (excluding stable statin therapy, as noted above), amphetamines and amphetamine derivatives, weight loss medications or supplements, corticosteroids, anticonvulsants, human immunodeficiency virus protease inhibitors, anticoagulants, and cyclosporine. 
The primary endpoint of this study was the percentage change in LDL cholesterol levels from baseline to week 8 during Period II comparing colesevelam with placebo. Secondary endpoints included the change in LDL cholesterol levels from week 8 to week 26 and from baseline to week 26 (Periods II and III), change in HDL and non-HDL cholesterol levels, triglyceride levels, TC, and ApoA-I and ApoB throughout Periods II and III, as well as the number of patients achieving an LDL cholesterol level of $<110 \mathrm{mg} / \mathrm{dL}$ by the end of Period III. Efficacy of all treatment outcomes was evaluated using intent-to-treat analysis, including all randomized patients with at least one lipid measurement beyond baseline, and who had received at least one dose of study drug. Last observation carried forward analysis was used to account for any missing efficacy measurements for both the week 8 and week 26 assessments. All adverse drug events (ADRs) and/or changes in subject physical characteristics were evaluated continuously through the study periods, and classified by both severity and likely relation to the study medication.

The initial subject pool of 294 was screened for inclusion, and 194 subjects were randomized to receive placebo $(n=65)$, colesevelam $1.875 \mathrm{~g} /$ day $(\mathrm{n}=65)$, or colesevelam $3.75 \mathrm{~g} /$ day $(\mathrm{n}=64)$. Completion of Period II was achieved by 186 subjects, while completion of the entire 26-week study was achieved by 173 subjects. Baseline characteristics between the study groups were similar. Compliance with the study medication during Period II, based on pill counting of unused tablets, was $85.5 \%$ in the placebo group, $85.3 \%$ in the colesevelam $1.875 \mathrm{~g} /$ day group, and $87.3 \%$ in the colesevelam $3.75 \mathrm{~g} /$ day group. Stable statin therapy was documented in 47 subjects during Period II, and was continued in these subjects during Period III.

After the end of the initial treatment period (Period II, baseline to week 8 ), the colesevelam $3.75 \mathrm{~g} /$ day group was found to have a reduction in LDL cholesterol levels of $12.5 \%$ $(P<0.001)$ and the colesevelam $1.875 \mathrm{~g} /$ day group had a LDL cholesterol level reduction of $6.3 \%(P<0.031)$, when compared with placebo. When broken into statin-naïve and statin-experienced subjects, the reductions were $10.6 \%$ $(P<0.001)$ and $20.2 \%(P<0.031)$, respectively. Reductions were not significantly associated with subject gender, age, body mass index, or the dosing schedule used (once versus twice daily). The colesevelam $3.75 \mathrm{~g} /$ day group also experienced a significant reduction in TC $(7.4 \% ; P=0.001)$, non-HDL cholesterol levels $(10.9 \% ; P=0.0001)$, and ApoB

\begin{tabular}{|c|}
\hline $\begin{array}{c}\text { Subjects screened for inclusion } \\
\mathrm{n}=247\end{array}$ \\
\hline $\mathrm{I}$ \\
\hline $\begin{array}{c}\text { Subjects randomized for inclusion } \\
\mathrm{n}=194\end{array}$ \\
\hline $\mathrm{I}$
\end{tabular}

Period II: Baseline to week 8

\begin{tabular}{|c|c|c|}
\hline $\begin{array}{c}\text { Placebo } \\
\mathrm{n}=65\end{array}$ & $\begin{array}{c}\text { Colesevelam } 1.875 \text { g/day group } \\
\mathrm{n}=65\end{array}$ & $\begin{array}{c}\text { Colesevelam } 3.75 \text { g/day group } \\
\mathrm{n}=64\end{array}$ \\
\hline $\mathrm{I}$ & $\mathrm{I}$ & $\mathrm{I}$ \\
\hline Withdrawals & Withdrawals \\
$\mathrm{n}=1$ & $\mathrm{n}=5$ & Withdrawals \\
$\mathrm{n}=2$
\end{tabular}

Period III: Week 8 to week 26

\begin{tabular}{|c|c|c|}
\hline $\begin{array}{c}\text { Placebo } \\
\mathrm{n}=64\end{array}$ & $\begin{array}{l}\text { Colesevelam } 1.875 \mathrm{~g} / \text { day group } \\
\qquad \mathrm{n}=60\end{array}$ & $\begin{array}{l}\text { Colesevelam } 3.75 \mathrm{~g} / \text { day group } \\
\qquad \mathrm{n}=62\end{array}$ \\
\hline 1 & 1 & 1 \\
\hline $\begin{array}{l}\text { Withdrawals } \\
\quad n=5\end{array}$ & $\begin{array}{l}\text { Withdrawals } \\
\qquad n=6\end{array}$ & $\begin{array}{l}\text { Withdrawals } \\
\quad n=2\end{array}$ \\
\hline
\end{tabular}

Completion: Baseline to week 26

\begin{tabular}{|c|c|c|}
\hline $\begin{array}{c}\text { Placebo } \\
\mathrm{n}=59\end{array}$ & Colesevelam 1.875 g/day group & $\begin{array}{c}\text { Colesevelam } 3.75 \text { g/day group } \\
\mathrm{n}=54\end{array}$ \\
\hline
\end{tabular}

Figure 2 Subject randomization and completion from baseline to Period III completion. Adapted with permission from Stein et al. ${ }^{35}$ 
(8.3\%; $P=0.0009)$. The colesevelam $3.75 \mathrm{~g} /$ day group experienced a significant increase in both HDL cholesterol levels $(6.1 \% ; P=0.008)$ and ApoA-I $(6.9 \% ; P=0.006)$. While not significant, a $5.1 \%$ increase in triglyceride levels occurred in the colesevelam $3.75 \mathrm{~g} /$ day group when compared with placebo.

During the open-label Period III of the trial, a significant reduction in LDL cholesterol of $9.3 \%(P<0.001)$ was found by week 26 . When analyzed further, the reductions in LDL cholesterol were as follows: the Period II placebo group experienced a reduction of $14.5 \%(P<0.001)$; the Period II colesevelam $1.875 \mathrm{~g} /$ day group experienced a reduction of $11.6 \%$ ( $P<0.001)$; and the Period II colesevelam $3.75 \mathrm{~g} /$ day group experienced a reduction of $1.9 \%(P=0.482)$.

Overall, colesevelam $3.75 \mathrm{~g} /$ day therapy during this clinical trial had several significant impacts on both primary and secondary endpoints. A reduction of LDL cholesterol as the primary efficacy endpoint was $14.0 \%(P<0.001)$ for the full duration of the trial. Colesevelam $3.75 \mathrm{~g} /$ day also reduced TC by $8.0 \%(P<0.001)$, non-HDL cholesterol levels by $11.3 \%$ ( $P<0.001)$, and ApoB by $11.3 \%(P<0.001)$ when evaluated for the full duration of the trial. Increases in HDL cholesterol were $8.1 \%(P<0.001)$ and increases in ApoA-I were $5.6 \%(P<0.001)$, while triglycerides, when looked at in relation to the full duration of the trial, increased significantly by $11.5 \%(P<0.001)$.

Finally, seven subjects achieved the clinically significant endpoint of LDL cholesterol of $<110 \mathrm{mg} / \mathrm{dL}$ during Period II, of whom five were in the colesevelam $3.75 \mathrm{~g} /$ day arm and two were in the $1.875 \mathrm{~g} /$ day arm. When looking at combined statin use, two subjects were statin-naïve and five were statinexperienced. Completion of Period III resulted in 14 subjects achieving LDL cholesterol of $<110 \mathrm{mg} / \mathrm{dL}$. Of these subjects, four were in the colesevelam $3.75 \mathrm{~g} /$ day arm in Period II, seven were in the colesevelam $1.875 \mathrm{~g} /$ day arm in Period II, and three were in the placebo arm in Period II. When looking further into statin use in this subset of subjects, seven were statin-naïve and seven were statin-experienced.

\section{Adverse effects and tolerability}

Overall, the study data to date have shown colesevelam to be well tolerated in 10-17 year old patients. Not surprisingly, the most commonly reported ADRs were gastrointestinal in nature, including abdominal pain, diarrhea, nausea, and vomiting. In the randomized, double-blind, eight-week study and 18-week open-label study periods performed in 194 children aged 10-17 years with $\mathrm{HeFH}, 6.3 \%$ of the patients receiving colesevelam $3.75 \mathrm{~g} /$ day experienced one or more ADRs, compared with $10.8 \%$ of the patients on colesevelam $1.875 \mathrm{~g}$ /day and $10.8 \%$ on placebo (see Table 1). Nasopharyngitis, upper respiratory tract infection, and headache were the ADRs experienced by $\geq 2 \%$ of subjects compared with placebo. Patient compliance during the study was $87.3 \%$ (colesevelam $3.75 \mathrm{~g} /$ day), $85.3 \%$ (colesevelam $1.875 \mathrm{~g} /$ day), and $85.5 \%$ (placebo). All of the subjects completing the study had the normal changes in both weight and height expected for normal maturation. Data regarding ADRs associated with the long-term use of colesevelam in adolescents are lacking, so further investigation is needed.

\section{Contraindications and precautions Triglycerides}

Colesevelam can increase serum triglyceride levels. An elevation in serum triglyceride levels increases the risk of pancreatitis. The manufacturer indicates that the use of colesevelam is contraindicated in patients with serum triglyceride levels $>500 \mathrm{mg} / \mathrm{dL}$ or a history of triglycerideinduced pancreatitis. ${ }^{28}$ According to the NCEP, colesevelam is relatively contraindicated in patients with serum triglyceride levels $>200 \mathrm{mg} / \mathrm{dL}$ and absolutely contraindicated when triglycerides exceed $400 \mathrm{mg} / \mathrm{dL}$, or if there is a diagnosis of familial dysbetalipoproteinemia. ${ }^{36}$ These recommendations are for the general adult population. In children it may be prudent to use the more conservative NCEP recommendations to reduce the risk of complications.

Due to the ability of colesevelam to bind cholesterol, it may also bind some medications affecting gastrointestinal absorption. Patients with a deficit in levels of fat-soluble vitamins (A, D, E, and $\mathrm{K}$ ) should not take colesevelam.

Table I Adverse reactions reported in $\geq 2 \%$ of patients in placebo-controlled clinical study of colesevelam as monotherapy or combination therapy with a stable statin regimen

\begin{tabular}{|c|c|c|c|}
\hline & \multicolumn{2}{|c|}{ Number of subjects (\%) } & \multirow[b]{2}{*}{$\begin{array}{l}\text { Period III } \\
\text { colesevelam } \\
(n=I 84)\end{array}$} \\
\hline & $\begin{array}{l}\text { Period II } \\
\text { colesevelam } \\
(n=129)\end{array}$ & $\begin{array}{l}\text { Period II } \\
\text { placebo } \\
(n=65)\end{array}$ & \\
\hline Nasopharyngitis & $8(6.2)$ & $3(4.6)$ & $10(5.4)$ \\
\hline Fatigue & $5(3.9)$ & $\mathrm{I}(\mathrm{I} .5)$ & $0(0.0)$ \\
\hline Headache & $5(3.9)$ & $2(3.1)$ & $14(7.6)$ \\
\hline $\begin{array}{l}\text { Upper respiratory tract } \\
\text { infection }\end{array}$ & $2(1.6)$ & $3(4.6)$ & $9(4.9)$ \\
\hline Gastroenteritis & $2(1.6)$ & $0(0.0)$ & $4(2.2)$ \\
\hline Influenza & $2(1.6)$ & $0(0.0)$ & $7(3.8)$ \\
\hline Nausea & $2(1.6)$ & $0(0.0)$ & $7(3.8)$ \\
\hline Abdominal pain & $2(1.6)$ & $0(0.0)$ & $6(3.3)$ \\
\hline Pharyngolaryngeal pain & $2(1.6)$ & $0(0.0)$ & $6(3.3)$ \\
\hline
\end{tabular}


When administered with colesevelam, the effectiveness of multivitamins, warfarin, levothyroxine, digoxin, phenytoin, and the ethinyl estradiol and norethindrone components of many oral contraceptives comes into question. It is recommended that these medications, as well as any medication with a narrow therapeutic index, are dosed at least four hours prior to colesevelam administration.

One well known side effect of colesevelam is constipation. Patients should not take colesevelam if they have a history of bowel obstruction, gastroparesis, or other gastrointestinal motility issues. A history of chronic constipation can increase the risk of bowel obstruction.

\section{Special considerations}

Patients who have difficulty swallowing or are at risk of choking should not take the tablet form of colesevelam. A powder form is available that is made into a suspension. The suspension can be used in patients who have difficulty swallowing pills. Data are lacking on the safety of the suspension in children and adolescents.

Colesevelam is a Pregnancy Category B drug. To date, no well controlled studies have been conducted in pregnant women. During pregnancy there is an increased need for vitamins and minerals. Absorption of vitamins and minerals, especially fat-soluble vitamins, may be decreased with colesevelam. In animal studies of rats and rabbits no negative effect on the fetus was seen. Therefore, colesevelam should not be used in pregnancy unless the benefits of therapy clearly outweigh the risks. ${ }^{28}$

Due to the lack of systemic absorption of colesevelam, there is no need to adjust the dose for patients with minor renal or hepatic impairment. However, there are no good studies to show how patients respond with severe renal dysfunction (creatinine clearance $<30 \mathrm{~mL} / \mathrm{min}$ ). Theoretically there should be little to no impact on renal function, but due to the lack of relevant data, patients with severe renal dysfunction should not take colesevelam. ${ }^{28}$

Colesevelam packets contain $24 \mathrm{mg}$ and $48 \mathrm{mg}$ of phenylalanine in the $1.875 \mathrm{~g}$ and $3.75 \mathrm{~g}$ dosage form, respectively. Phenylketonurics should therefore only consume the tablets. $^{28}$

\section{Dosage and administration}

Colesevelam is available in two different forms for oral administration, ie, $625 \mathrm{mg}$ tablets and suspensions available in $1.875 \mathrm{mg}$ and $3.75 \mathrm{mg}$ packets. The recommended dose is $3.75 \mathrm{~g} /$ day (six tablets or suspension packet) taken either once daily or divided into two doses of $1.875 \mathrm{~g}$ (three tablets or suspension packet). To prepare the suspension, the patient should be instructed to empty the contents of the packet into a glass and mix with 4-8 ounces of water. The solution needs to be stirred prior to administration. To decrease the incidence of esophageal distress, the suspension should not be taken unless it has been mixed with 4-8 ounces of water. Colesevelam should be taken along with a meal and liquid. ${ }^{28}$

Timing of doses is important when the patient is on multiple medications. Colesevelam may decrease the absorption of some medications (eg, multivitamins), and should be administered at least four hours after other medications, to decrease the risk of possible interactions. If a patient takes medications or vitamins in the morning with breakfast, then it would be prudent to administer the colesevelam either once daily with dinner or to split the dose between lunch and dinner. Any medications with a narrow therapeutic index should be monitored closely when initiating colesevelam. ${ }^{28}$

\section{Conclusion}

Bile acid sequestrants have long been considered an appropriate therapy for treatment of hypercholesterolemia in children and adolescents. Their safe and effective use as a class has been documented in the literature, and the mild ADR profile makes them a preferred choice for both compliance and efficacy. The recent clinical trial investigating the efficacy and safety of colesevelam in the child/adolescent population illustrates the significant impact that colesevelam, a selective, nonsystemic bile acid sequestrant, can have on the lipid profile of a patient, while maintaining a low incidence of ADRs. ${ }^{35}$ The recent approval of a powder formulation in the US for treatment of HeFH improves the ability to administer colesevelam effectively, and with better adherence and compliance in an at-risk population. While further trials would be beneficial to determine any long-term complications that may arise with colesevelam therapy, such as the risk of fatsoluble vitamin depletion, the currently available data and historic use of bile acid sequestrants as a class suggest that the use of colesevelam in the pediatric or adolescent $\mathrm{HeFH}$ patient population is appropriate.

\section{Disclosure}

The authors report no conflict of interest in this work.

\section{References}

1. Cox E, Halloran D, Homan S, Welliver S, Mager D. Trends in the prevalence of chronic medication use in children: 2002-2005. Pediatrics. 2008;122(5):e1053-e1061. 
2. McGill H, McMahan C, Zieske A, et al. Associations of coronary heart disease risk factors with the intermediate lesion of atherosclerosis in youth. The Pathobiological Determinants of Atherosclerosis in Youth Research Group. Arterioscler Thromb Vasc Biol. 2000;20: 1998-2004.

3. Berenson G, Srinivasan S, Newman W, Tracy R, Wattigney W. Association between multiple cardiovascular risk factors and atherosclerosis in children and young adults. The Bogalusa Heart Study. N Engl J Med. 1998;338(23):1650-1656.

4. Daniels S, Greer F; Committee on Nutrition. Lipid screening and cardiovascular health in childhood. Pediatrics. 2008;122:198-208.

5. Raitakari O, Juonala M, Kahonen M, et al. Cardiovascular risk factors in childhood and carotid artery intima-media thickness in adulthood: The cardiovascular risk in young Finns study. JAMA. 2003;290(17): 2277-2283.

6. Srinivasan SR, Frontini MG, Xu J, Berenson G. Utility of childhood nonhigh-density lipoprotein cholesterol in predicting adult dyslipidemia and other cardiovascular risk: the Bogalusa Heart Study. Pediatrics. 2006;118(1):201-206.

7. Tonstad S. Role of lipid-lowering pharmacotherapy in children. Paediatr Drugs. 2000;2(1):11-22.

8. Shafiq N, Singh M, Kaur S, Khosla P, Malhotra S. Dietary treatment for familial hypercholesterolaemia. Cochrane Database Syst Rev. 2010;1: CD001918.

9. Ohshiro T, Shimabukuro T, Sunagawa M, Ohta T. An 11-year-old boy with familial hypercholesterolemia showing multiple xanthomas and advanced atherosclerosis, who responded to lipid-lowering therapy using statin. J Atheroscler Thromb. 2009;16(5):698-701.

10. Strong J, Malcolm G, Newman W, Oalmann M. Early lesions of atherosclerosis in childhood and youth: Natural history and risk factors. $\mathrm{J} \mathrm{Am}$ Coll Nutr. 1992;11:S51-S54.

11. Newman W, Freedman D, Voors A, et al. Relation of serum lipoprotein levels and systolic blood pressure to early atherosis: The Bogalusa Heart Study. N Engl J Med. 1986;314(3):138-144.

12. Sallustio F, Rotondo F, Di Legge S, Stanzione P. Cilostazol in the management of atherosclerosis. Curr Vasc Pharmacol. 2010;8(3):363-372.

13. Robless P, Mikhailidis D, Stansby G. Cilostazol for peripheral arterial disease. Cochrane Database Syst Rev. 2008;1:CD003748.

14. Wray R, Neil H, Rees J. Screening for hyperlipidaemia in childhood. Recommendations of the British Hyperlipidaemia Association. J R Coll Physicians Lond. 1996;30(2):115-118.

15. van der Graaf A, Kastelein J, Wiegman A. Heterozygous familial hypercholesterolemia in childhood: Cardiovascular risk prevention. J Inherit Metab Dis. 2009;32(6):699-705.

16. Guardamagna O, Abello F, Saracco P, Baracco V, Rolfo E, Pirro M. Endothelial activation, inflammation and premature atherosclerosis in children with familial dyslipidemia. Atherosclerosis. 2009;207(2): $471-475$.

17. Mabuchi H, Koizumi J, Shimizu M, Takeda R. Development of coronary heart disease in familial hypercholesterolemia. Circulation. 1989;79(2): 225-232.

18. Martino F, Loffredo L, Carnevale R, et al. Oxidative stress is associated with arterial dysfunction and enhanced intima-media thickness in children with hypercholesterolemia: The potential role of nicotinamideadenine dinucleotide phosphate oxidase. Pediatrics. 2008;122(3): e648-e655.
19. Li S, Chen W, Srinivasan S, et al. Childhood cardiovascular risk factors and carotid vascular changes in adulthood: The Bogalusa Heart Study. JAMA. 2003;290(17):2271-2276.

20. American Academy of Pediatrics. Cardiovascular risk reduction in high-risk pediatric populations. Pediatrics. 2007;119(3):618-621.

21. de Jongh S, Lilien M, op’t Roodt J, Stroes E, Kastelein J. Early statin therapy restores endothelial function in children with familial hypercholesterolemia. J Am Coll Cardiol. 2002;40(12):2117-2121.

22. Ballantyne C, Herd J, Dunn J, Jones P, Farmer J, Gotto A. Effects of lipid lowering therapy on progression of coronary and carotid artery disease. Curr Opin Lipidol. 1997;8(6):354-361.

23. Vuorio A, Kovanen P, Gylling H. Hypolipidemic treatment of familial heterozygous hypercholesterolemia: A lifelong challenge. Expert Rev Cardiovasc Ther. 2004;2(3):405-415.

24. Insull W, Toth P, Mullican W, et al. Effectiveness of colesevelam hydrochloride in decreasing LDL cholesterol in patients with primary hypercholesterolemia: A 24-week randomized controlled trial. Mayo Clin Proc. 2001;76(10):971-982.

25. Hunninghake D, Insull W, Toth P, Davidson D, Donovan J, Burke S. Coadministration of colesevelam hydrochloride with atorvastatin lowers LDL cholesterol additively. Atherosclerosis. 2001;158(2):407-416.

26. Knapp H, Schrott H, Ma P, et al. Efficacy and safety of combination simvastatin and colesevelam in patients with primary hypercholesterolemia. Am J Med. 2001;110(5):352-360.

27. Davidson M, Toth P, Weiss S, et al. Low-dose combination therapy with colesevelam hydrochloride and lovastatin effectively decreases low-density lipoprotein cholesterol in patients with primary hypercholesterolemia. Clin Cardiol. 2001;24(6):467-474.

28. Colesevelam hydrochloride $\left(\right.$ Welchol $\left.^{\circledR}\right)$ [package insert]. Parsippany, NJ: Daiichi Sankyo, Inc; 2009.

29. Ast M, Frishman W. Bile acid sequestrants. J Clin Pharmacol. 1990; 30(2):99-106.

30. Shepherd J. Mechanism of action of bile acid sequestrants and other lipid-lowering drugs. Cardiology. 1989;76 Suppl 1:65-74.

31. LaRosa J. Review of clinical studies of bile acid sequestrants for lowering plasma lipid levels. Cardiology. 1989;76 Suppl 1:55-64.

32. Heller D, Burke S, Davidson D, Donovan J. Absorption of colesevelam hydrochloride in healthy volunteers. Ann Pharmacother. 2002;36(3): 398-403.

33. Rosebaum D, Petersen J, Ducharme S, Markham P, Goldberg D. Absorption, distribution, and excretion of GT31-104, a novel bile acid sequestrant, in rats and dogs after acute and subchronic administration. J Pharm Sci. 1997;86(5):591-595.

34. Ose L, MacMahon O, Theisen K, et al. Once per day and split dosing of colesevelam in patients with Type IIa hypercholesterolemia [abstract]. Presented at: 5th International Symposium on Multiple Risk Factors in Cardiovascular Disease: Global Assessment and Intervention. Venice, Italy; 1999, Oct 28-31.

35. Stein E, Marais A, Szamosi T, et al. Colesevelam hydrochloride: Efficacy and safety in pediatric subjects with heterozygous familial hypercholesterolemia. J Pediatr. 2010;156(2):231-236.

36. Expert Panel on Detection, Evaluation, and Treatment of High Blood Cholesterol in Adults. Executive Summary of The Third Report of The National Cholesterol Education Program (NCEP) Expert Panel on Detection, Evaluation, and Treatment of High Blood Cholesterol in Adults (Adult Treatment Panel III). JAMA. 2001;285(19):2486-2497.
Adolescent Health, Medicine and Therapeutics

\section{Publish your work in this journal}

Adolescent Health, Medicine and Therapeutics is an international, peer-reviewed, open access journal focusing on health, pathology, and treatment issues specific to the adolescent age group. All aspects of health maintenance, preventative measures and disease treatment interventions are addressed within the journal and practitioners from

\section{Dovepress}

all disciplines are invited to submit their work as well as healthcare researchers and patient support groups.. The manuscript management system is completely online and includes a very quick and fair peerreview system. Visit http://www.dovepress.com/testimonials.php to read real quotes from published authors. 